\title{
Microlens array production in a microtechnological dry etch and reflow process for display applications
}

\section{T. Knieling}

thomas.knieling@isit.fraunhofer.de

\section{Shafi}

\section{W. Lang}

\section{W. Benecke}

Fraunhofer Institut für Siliziumtechnologie (ISIT), Fraunhoferstrasse 1, 25524 Itzehoe, Germany

Institut für Mikrosensoren, -aktuatoren und -systeme (IMSAS), University of Bremen, Otto-HahnAllee, 28359 Bremen, Germany

Institut für Mikrosensoren, -aktuatoren und -systeme (IMSAS), University of Bremen, Otto-HahnAllee, 28359 Bremen, Germany

Fraunhofer Institut für Siliziumtechnologie (ISIT), Fraunhoferstrasse 1, 25524 Itzehoe, Germany

The fabrication of arrays consisting of densely ordered circular convex microlenses with diameters of $126 \mu \mathrm{m}$ made of quartz glass in a photoresist reflow and dry etch structure transition process is demonstrated. The rectangular lens arrays with dimensions of $6 \mathrm{~mm} \times 9 \mathrm{~mm}$ were designed for focussing collimated light on the pixel center regions of a translucent interference display, which also was produced in microtechnological process steps. The lenses focus light on pixel centers and thus serve for increasing display brightness and contrast since incoming collimated light is partially blocked by opaque metallic ring contacts at the display pixel edges. The focal lengths of the lenses lie between $0.46 \mathrm{~mm}$ and $2.53 \mathrm{~mm}$ and were adjusted by varying the ratio of the selective dry etch rate of photoresist and quartz glass. Due to volume shrinking and edge line pinning of the photoresist structures the lenses curvatures emerge hyperbolic, leading to improved focussing performance. [DOI: http://dx.doi.org/10.2971/jeos.2012.12007]

Keywords: microlens array, quartz glass, microstructures, light focussing, brightness enhancement, interference display

\section{INTRODUCTION}

Many displays consist of pixels which have to be separated laterally due to electrical or mechanical reasons. In direct view or during projection pixel spaces can cause disturbing effects like the screen door effect or Moirè patterns [1]. During former activities microstructured translucent interference display was build for structured illumination for topographic surface analysis [2,3]. The pixels with diameters of $126 \mu \mathrm{m}$ are realized by electrostatically deflectable membranes consisting of dielectric layer stacks with adjusted thicknesses and dispersions. Electrostatic forces between upper and lower metallic ring contacts cause membrane deflection in order to achieve

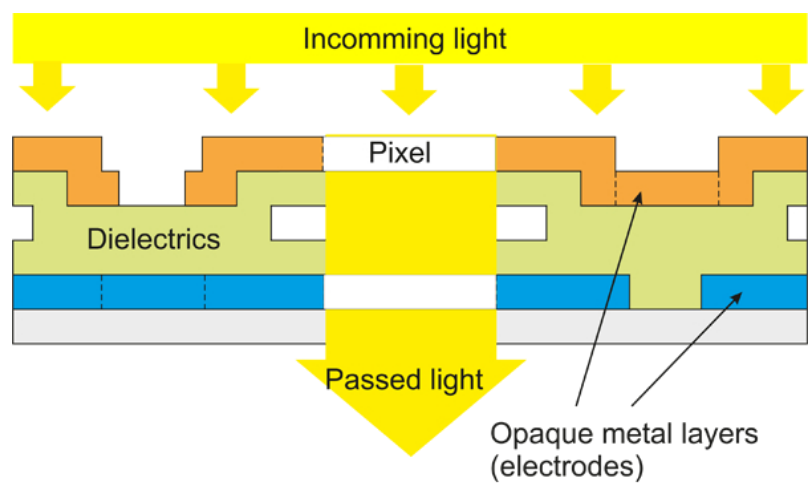

FIG. 1 Interference display assembly. Since incoming light is blocked by metallic ring electrodes, a lens array for light focussing on the pixel center areas will be developed. certain spatial and incoherent color or brightness modulation due to interference effects (Figure 1).

Due to the shading of the metallic contacts the active pixel area is reduced to $42,4 \%$. Since there are still not used spaces between densely ordered circular pixels shapes, the light blocking area referred to the whole display area ist reduced, but still $36 \%$. The aim of the monolithic microlens array is to collect the light from the metallic contact regions into the pixel center areas. Moreover light which is simply transmitted unmodulated through the passive spaces between pixels will be blocked by an opaque chrome aperture array.

\section{MICROLENS ARRAY PRODUCTION}

The production process is basing on a resist reflow and a lens thickness scaling pattern transfer process [4]. Figure 2 shows schematically the process flow.

Since lens and pixel diameters are the same, also same lithographic masks for UV light illumination as for display production were used for all lithographic steps. The substrates consist of 4 inch quartz glass wafers with thicknesses of 525 $\mu \mathrm{m}$. At first circular cylinders with heights of about $200 \mathrm{~nm}$ were patterned in a dry reactive ion etch (RIE) process with Fluorine gas. In the next step these structures serve as capillary barriers for pinning the viscous photoresist at the cylinder 


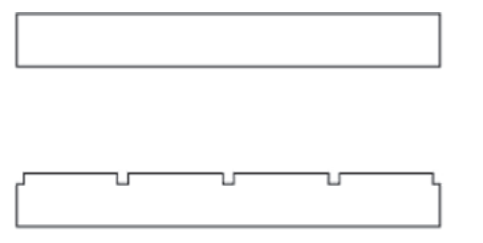

Quartz substrate

Patterning of pinning structures (lithography, $200 \mathrm{~nm}$ dry etching (RIE))

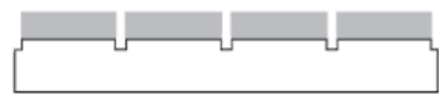

Resist cylinders for reflow prozess, $3,9 \mu \mathrm{m}$

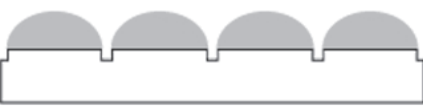

Reflow step $180^{\circ} \mathrm{C}, 10 \mathrm{Min}$.

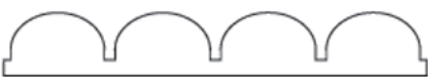

Structure transition by dry etching (RIE)

Aperture patterning

(sputtered $\mathrm{Cr}, 80 \mathrm{~nm}$ )

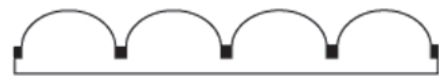

FIG. 2 Microlens array and interference display assembly.
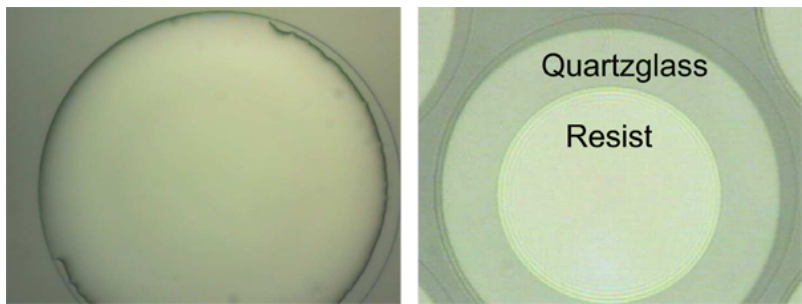

FIG. 3 Left: Partially pinned reflow resist due to slight UV overexposing. Right: Partially processed structure transfer.

edges during reflow. The reflow process was conducted with 3,9 $\mu \mathrm{m}$ thick patterned cylinders consisting of conventional AZ1518 resist provided by MicroChemicals. A challenge here is to adjust UV illumination parameters (time, power, mask mismatch) well in order to get the both (glass and resist) cylinders fully matched. Moreover the resist cylinders diameters was increased by a few $\mu \mathrm{m}$ to get the pinning edges covered along the whole circle edge line. The resist reflow temperature was $170{ }^{\circ} \mathrm{C}$ for $5 \mathrm{~min}$. on a hotplate. During the reflow the the resist becomes viscous and solution parts evaporate out the resist, leading to a certain volume shrink. The result is a lens like resist formation with pinned edges and hyperbolic surface profile (see Figure 3, left, and Figure 7 in Section 3).

The resists structures are subsequently being transferred into the quartz glass substrate by a dry RIE process (Figure 3, right). The Figure 4 shows surface topology of an array segment which was measured by laser scanning profilometry.

The dry etch parameters determine the lens thicknesses and thus their focal lengths directly (Table 1).

A wet etch step with buffered oxide etch solution was applied in order to reduce lens surface roughness which especially occurred at the lens edges during dry etch pattern transfer. Figure 4 shows the resulting surface topology of an array segment which was measured by laser scanning profilometry.

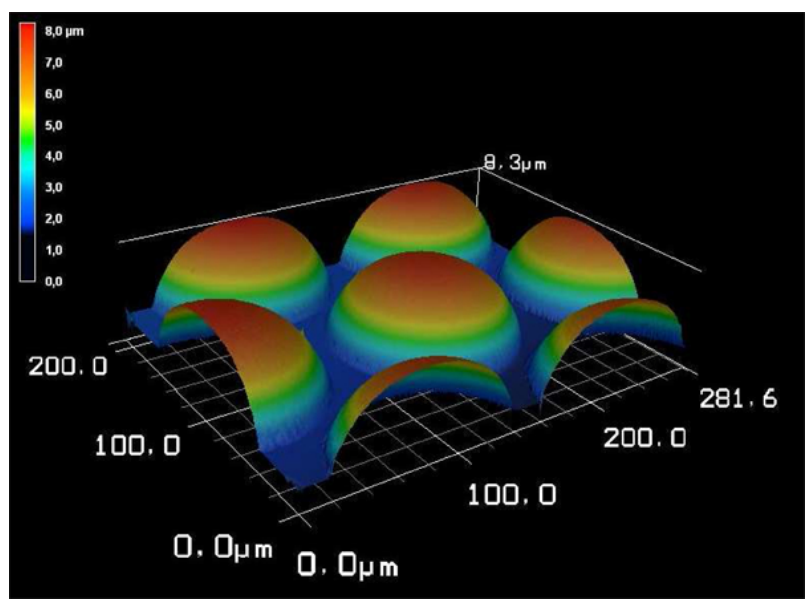

FIG. 4 Lens profiles analyzed with laser scanning profilometry. The obtained surface roughness is $R_{z}=3.00 \mu \mathrm{m}$ and $R_{a}=0.57 \mu \mathrm{m}$, respectively.

\begin{tabular}{|c|c|c|}
\hline Etch parameters & $t_{l}[\mu \mathrm{m}]$ & $f[\mathrm{~mm}]$ \\
\hline $\begin{array}{c}10 \mathrm{sccm} \mathrm{SF}_{6} \\
10 \mathrm{sccm} \mathrm{\textrm {O } _ { 2 }}, \\
15^{\circ} \mathrm{C}, 100 \mathrm{mTorr}, \\
30 \mathrm{~W}, 40 \mathrm{~min} .\end{array}$ & 1.15 & 2.53 \\
\hline $\begin{array}{c}10 \mathrm{sccm} \mathrm{SF}_{6} \\
0 \mathrm{sccm} \mathrm{O} \\
15^{\circ} \mathrm{C}, 100 \mathrm{mTorr} \\
30 \mathrm{~W}, 70 \mathrm{~min} .\end{array}$ & 1.75 & 1.70 \\
\hline $\begin{array}{c}15 \mathrm{sccm} \mathrm{CHF}_{3}, \\
32 \mathrm{sccm} \mathrm{CF} \mathrm{CF}_{4} \\
15^{\circ} \mathrm{C}, 160 \mathrm{mTorr} \\
70 \mathrm{~W}, 100 \mathrm{~min} .\end{array}$ & 6.50 & 0.65 \\
\hline $\begin{array}{c}25 \mathrm{sccm} \mathrm{CHF}, \\
32 \mathrm{sccm} \mathrm{CF}_{4}, \\
5^{\circ} \mathrm{C}, 220 \mathrm{mTorr}, \\
100 \mathrm{~W}, 100 \mathrm{~min} .\end{array}$ & 9.35 & 0.48 \\
\hline $\begin{array}{c}30 \mathrm{sccm} \mathrm{CHF}{ }_{3}, \\
32 \mathrm{sccm} \mathrm{CF} 4 \\
55^{\circ} \mathrm{C}, 250 \text { mTorr, } \\
100 \mathrm{~W}, 200 \text { min. }\end{array}$ & 11.38 & 0.46 \\
\hline
\end{tabular}

TABLE 1 Dry etch parameters and resulting lens specifications due to different glassresist etch rate selectivities. $t_{l}$ : Lens thickness, $f$ : Focal length.

Since the BOE etch rate is low, the lens thickness was only affected insignificantly.

The last step is to create an array consisting of chrome apertures for blocking of unmodulated light which has passed through the spaces between the display pixels. The chrome layer with a thickness of $30 \mathrm{~nm}$ was deposited in a conventional magnetron sputtering process. The display mask for pixel generation was again used to pattern the apertures in common lithographic and wet etch step. At last the substrate was cut into rectangular array pieces $(6 \mathrm{~mm} \times 9 \mathrm{~mm})$ with a rotating diamond saw. In Figure 5) light and scanning electronic microscopy views of the produced arrays are shown. 


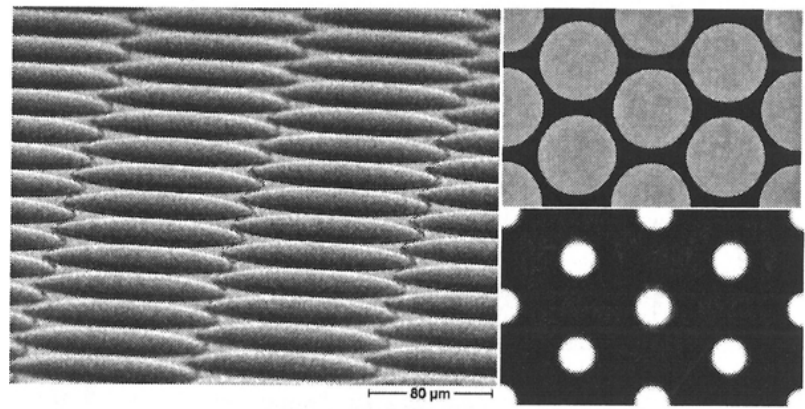

FIG. 5 Left: Tilted SEM view of a lens array. Upper right: Microscope view with focus in aperture plane. Lower right: Same view in focal plane.

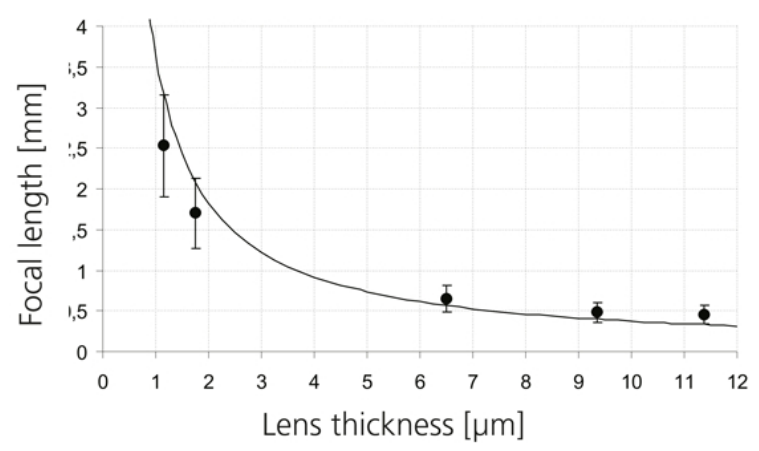

FIG. 6 Focal lengths of different lens variations. The continuous line shows the theoretical prediction for a lens radius of $63 \mu \mathrm{m}$ according to Eq. (1).

\section{MEASUREMENT RESULTS}

For thin (aspheric) lenses the following Eq. (1) can be used for calculating the focal length $f$ depending on the lens geometry and ist refractive index $n$ [5]:

$$
f=\frac{d_{\text {lens }}^{2}+r_{\text {lens }}^{2}}{2 d_{\text {lens }}(n-1)}
$$

$d_{\text {lens }}$ and $r_{\text {lens }}$ are the lens thickness and the lens radius, respectively.

The focal lengths were measured by focus variation, i.e. zaxis motion, in white light microscopy. Figure 6) shows good agreement between theoretical prediction and results of real lens analysis.

As it was mentioned, pinning structures, i.e. capillary barriers were chosen for obtaining aspheric lens profiles for improving light focussing efficiency, especially from the lens edge regions. For optical lenses usually the Zernike-polynomial or similar expressions are used to describe quantitatively the aspheric lens shape with the according Zernike coefficients [6]. Since the lenses produced here are not of optical quality, but nevertheless suited for this application, a reduced third order approach is used in this case:

$$
r=c_{2} \sqrt{\frac{d_{\text {lens }}^{2}-c_{3}^{2}}{c_{1}}-1}
$$

For allowing a comparison between the theoretical approximation (2) and real lens shapes they were measured with an

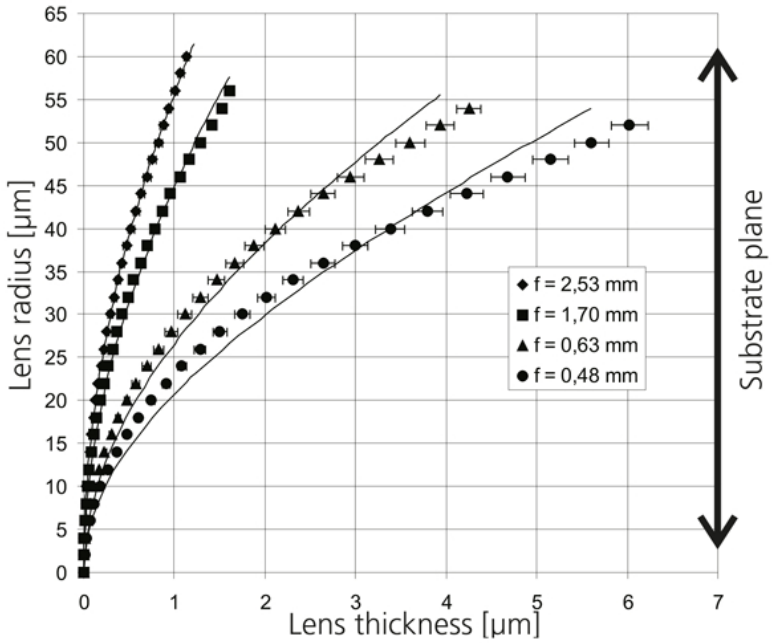

FIG. 7 Fitted lens profiles with hyperbolic approximation (2). Especially for thin lenses there is a good agreement between theoretical model (cont. lines) and the real shapes.

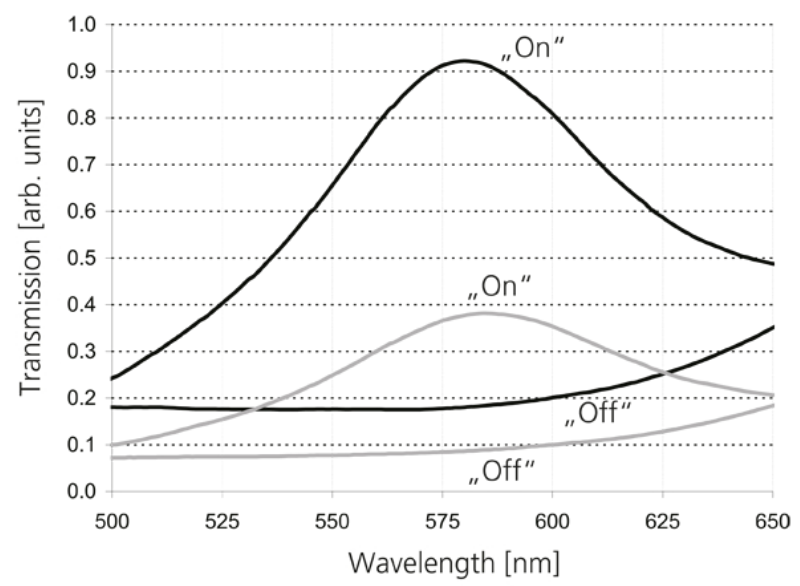

FIG. 8 Spectral curves with and without using light focussing lenses. The pixel efficiency is increased by a factor 2,64 .

AlphaStep profilometer. Figure 7) shows the results of this fitting, which was done withe the Origin software tool.

At last it is interesting to what extend the display pixel on/offefficiency has been increased by lens array implementation and also how the pixel contrast is influenced. For that light modulations along the optical paths of a display pixel with and without lens were compared by translucent spectral analysis $^{1}$. The result is shown in Figure 8. Surely both "on"- and "off" illumination intensities are increased to the same manner, since the lenses cannot distinguish between both states. Thus the pixel contrast remains nearly unaffected.

\section{ARRAY ASSEMBLY}

For protecting the lens surfaces the lens arrays were assembled in that way that the lens surfaces pointed towards the display pixels. Glass rods with diameters of the focal lengths were cut with a rotating diamond saw. Theses rods are serv-

\footnotetext{
${ }^{1}$ For this an Avantes Nanocalc spectrometer with spatially limited illumination by a glass fiber was used.
} 


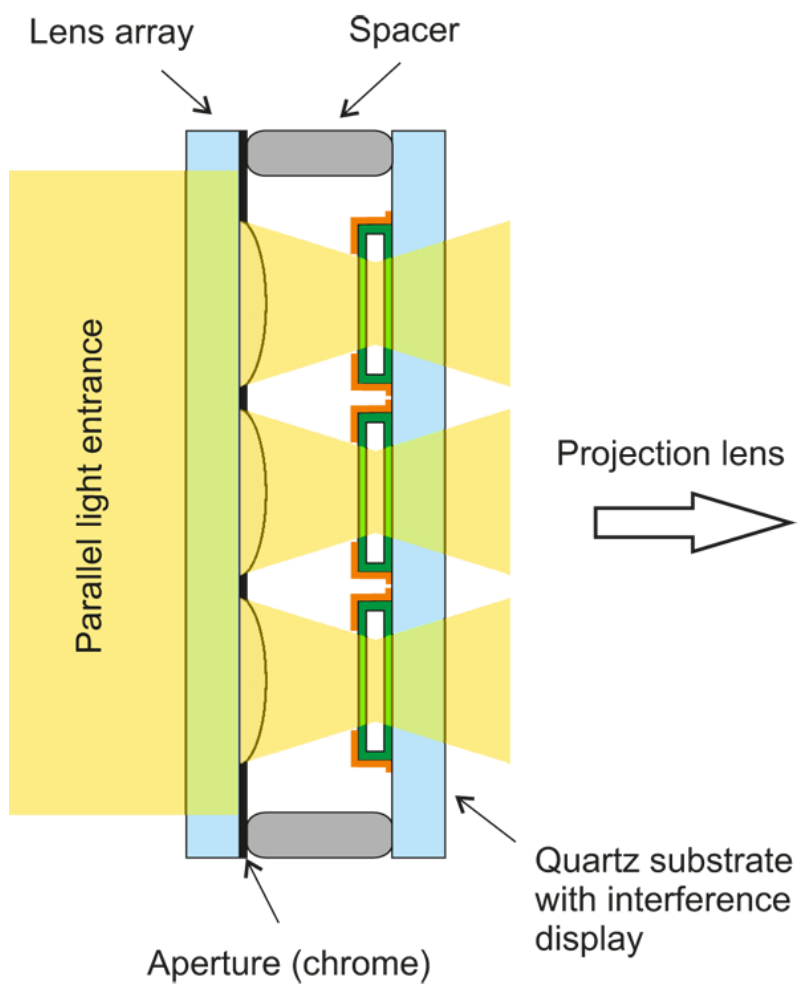

FIG. 9 Schematic setup and light paths in the lens array - interference display assembly.

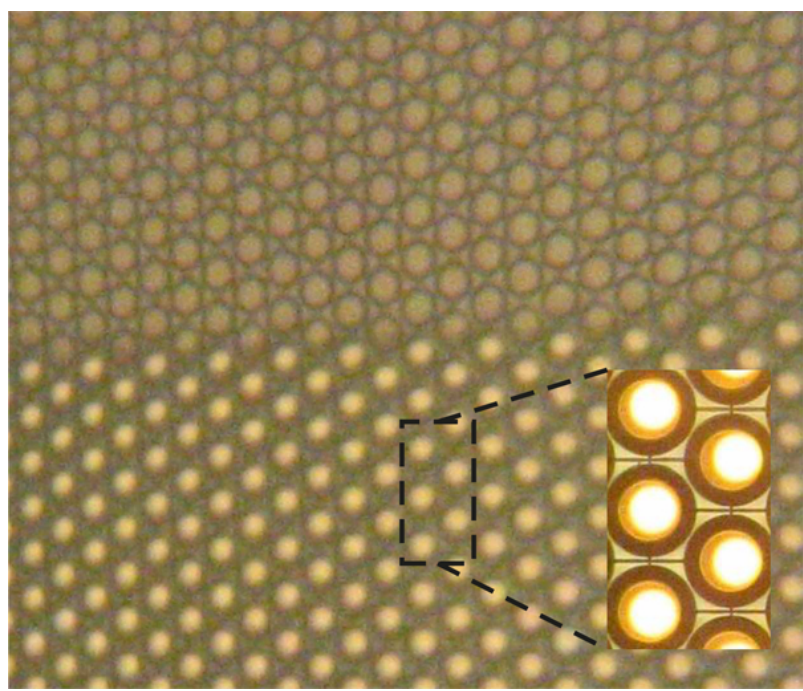

FIG. 10 Real lens array - interference display assembly. For demonstration purposes the upper display area is not covered by the lens array. The inset shows a magnified cutout where the pixel center areas are illuminated by focussed light coming from the array lenses. In this version the apertures on the lens array are missing.

ing as spacers between interference display and lens array. Epoxy hard glue was used for rod fixing. During glue pot life the relative position between display and lens array was adjusted with focus variation in a microscope. Figure 9) draws a schematic of the lens array-display assembly, Figure 10) shows pixel light focussing in a real setup containing a unaddressed display (all pixels show same color and grey level).

\section{CONCLUSION}

A simple, cost-effective dry etch and pattern transfer process is proven to be suited for producing monolithic glass lens arrays with process-dependent focal lengths. After assembling the lens array and the interference display an increase in pixel efficiency by a factor of 2.64 has been reached.

\section{ACKNOWLEDGEMENTS}

This work was funded by the German Ministry of Science and Education (BMBF) within the the project MicroEnd (16SV1222).

\section{References}

[1] E. Stupp, and M. Brennesholtz, Projection displays (Wiley-VCH, Weinheim, 1999).

[2] T. Knieling, M. Panitz, and W. Benecke, "Electrostatic actuated optical Fabry-Perot switches in passive matrix Displays," SPIE (5348-11), 1-11 (2004).

[3] T. Knieling, W. Lang, and W. Benecke, "Characteristics and electrical addressing of optical Fabry-Perot Displays," SPIE (5875-20), 1-10 (2005).

[4] Z. D. Popovic, R. A. Sprague, and G. A. N. Connel, "Technique for monolithic fabrication of microlens arrays," Appl. Optics 27 (7), 1281-1284 (1988).

[5] E. Hecht, Optik (3rd ed., Oldenbourg-Verlag, München/Wien, 2001).

[6] M. Born, and E. Wolf, Principles of Optics (Pergamon Press, 0xford, 1970). 